\title{
Control of Heme Metabolism with Synthetic Metalloporphyrins
}

\author{
Attallah Kappas and George S. Drummond \\ The Rockefeller University Hospital, New York 10021
}

\section{Introduction}

The red pigment heme plays a vital role in cellular metabolism, functioning as the prosthetic group of circulating (i.e., hemoglobin) and membrane-bound (i.e., cytochromes) hemeproteins concerned with oxygen transport, oxygen activation in chemical biotransformations, and electron transfer in cellular respiration and energy generation. Iron, the central metal atom of heme (Fig. 1), is a transition element and its ability to undergo reversible changes in oxidation state, $\mathrm{Fe}^{2+} \rightleftarrows \mathrm{Fe}^{3+}$, is essential to the participation of heme in physiological systems. Iron alone displays certain of the catalytic properties of heme, but chelation of the element by the protoporphyrin macrocycle and binding of the metal-porphyrin complex to specific apoproteins enhance the catalytic activities of the free metal ion by 5-10 orders of magnitude (1). The evolution of specific iron-porphyrin-protein complexes was a crucial process in providing developing organisms with the means by which the great versatility and adaptability of oxidative routes of metabolism could be utilized effectively.

There is a practical importance for clinicians to understand certain aspects of heme biology since the pathways by which heme is synthesized, utilized, or degraded to bile pigments are subject to genetic aberrations, which are manifest principally as the various forms of porphyria (2), as well as to environmental perturbations including those of an endocrine, nutritional, pharmacological, and toxicological nature. These factors, which are often under the control of, or need to be dealt with, by physicians and others involved in health care, influence significantly the biological impact of and individual adaptive responses to chemicals ranging from hormones to drugs and to toxic foreign agents, such as carcinogens and other environmental pollutants.

The major sites, in the liver, at which such perturbations in heme metabolism take place are at the levels of $\delta$-aminolevulinic acid (ALA) ${ }^{1}$ synthase and heme oxygenase (the rate-controlling enzymes in the synthesis of heme and in its catabolism to bile pigments, respectively) and at the formation and function of cytochrome(s) P-450, the family of monooxygenases which catalyzes the oxidative metabolism of numerous, structurally diverse, natural and foreign chemicals $(2,3)$. Pharmacologic factors which can lead to an enhancement or diminution of the formation or activities of these enzymes include therapeutic agents (4), environmental pollutants $(5,6)$, natural and synthetic hor-

Received for publication 8 July 1985.

1. Abbreviations used in this paper: ALA, $\delta$-aminolevulinic acid; T, testosterone.

\footnotetext{
J. Clin. Invest.

(c) The American Society for Clinical Investigation, Inc.

$0021-9738 / 86 / 02 / 0335 / 05 \$ 1.00$

Volume 77, February 1986, 335-339
}

mones (7-11), and the constituents of the human diet (12-14); among the latter are included not only the nutritive components of foods, such as proteins and carbohydrates, but numerous foreign substances of nonnutritive nature as well. Indeed, humans receive the bulk of their exposure to environmental pollutants through the diet (15) and the impact of many of these agents on the primary pathways of heme metabolism (Fig. 1) may be of considerable significance in human pathophysiology.

\section{Control of heme metabolism with synthetic metalloporphyrins}

Chemical perturbation of one or more of the primary pathways of heme metabolism has been widely used for experimental purposes in studies of heme biology; the majority of chemicals used for this purpose have been drugs, toxic organic substances, or comparable agents. However, apart from the limited use of heme itself to suppress exacerbations of the genetic disease, acute intermittent porphyria, which is an action attributed to heme repression of ALA-synthase formation in liver, there have been few investigations of the biological properties of other metalporphyrin complexes which might have clinically useful activities.

We have undertaken studies along these lines for the past several years and the results of this research provide clear evidence that certain synthetic heme analogues, in which the central iron atom is replaced by another element, possess biological properties which are qualitatively and quantitatively distinct from those of heme, and that it may be possible to utilize such synthetic metalloporphyrins for useful experimental and clinical purposes.

\section{Background}

In the decade (16) since it was first demonstrated in this laboratory that the essential trace element cobalt was a potent inducer of heme oxygenase in liver, numerous metals and metallic compounds (17) have been shown to also induce this liver enzyme and thus to enhance heme degradation, diminish cellular content of cytochrome P-450, and consequently, to impair cytochrome $\mathrm{P}-450$-dependent oxidative metabolism, which is a process primarily (though not exclusively) involved in overall chemical detoxification mechanisms. The metal compounds utilized in such studies have included inorganic elements such as cobalt, nickel, gold, platinum, chromium, tin, and mercury; organified metal species now widely used in agriculture; metal-containing drugs; and most recently, metals chelated with the same macrocycle, protoporphyrin IX, which comprises the ring structure of the natural metalloporphyrin, heme.

Among the inorganic elements that were capable of inducing heme oxygenase, tin proved to be unique in two respects; it induced the enzyme in the kidney rather than in liver, and its relative inducing potency was greater than that of any other 


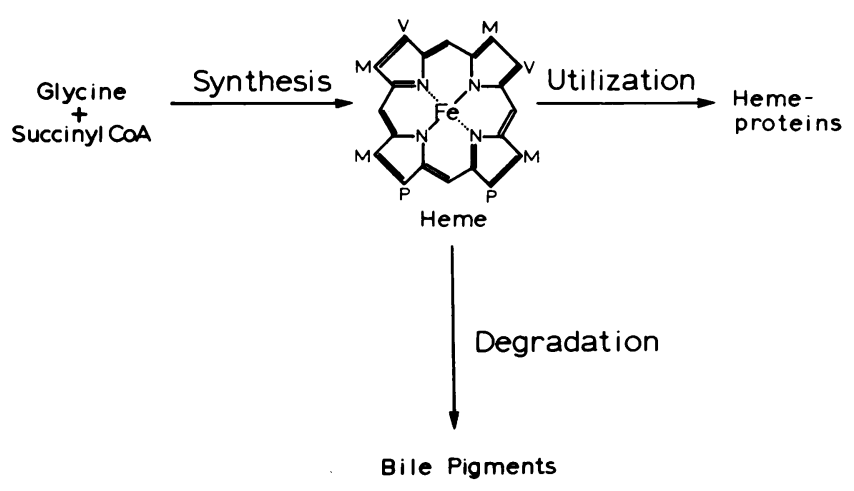

Figure 1. Primary pathways of heme metabolism.

comparable agent (18). Thus, tin, together with cobalt, provided distinctive metal species by which heme metabolism in renal and hepatic tissues could be independently explored. Furthermore, both elements are of considerable clinical and environmental medical interest; cobalt has been used for erythropoietic purposes in chronic renal disease (19), in the treatment of cyanide poisoning (20), and has been proposed for use as an antitumor agent $(21,22)$, while tin, in various organic forms, is now being widely employed as a biocidal agent for agricultural purposes $(23,24)$, a use which carries the potential of substantial human exposure to the compound.

In examining the actions of organic tin compounds on heme oxygenase, it became apparent that organification of the metal not only shifted the tissue site of action of the contained element from kidney (16) to liver $(25,26)$, a fact of considerable toxicological importance, but that it also greatly prolonged the enzyme-inducing action of the metal; for example, heme oxygenase induction persisted as long as $10 \mathrm{~d}$ after a single dose of an organotin compound. The incorporation of metals into various organic species thus had the potential to profoundly alter, both in degree and in site of action, the response of tissues, in terms of altered heme metabolism, to these elements.

This consideration prompted us to examine the possible consequences for heme-cytochrome P-450 metabolism, in the liver and other tissues, of incorporating such elements as tin and cobalt into the protoporphyrin macrocycle, and thus forming synthetic heme analogues. These synthetic metalloporphyrins would not be enzymatically degraded to bile pigments, since their central metal atoms, unlike the iron of heme, could not bind molecular oxygen. We anticipated therefore that the effects of such synthetic metalloporphyrins on heme-cytochrome P450 metabolism might be quantitatively or qualitatively different from those produced by the inorganic elements that they contained, or by heme itself. This anticipation proved to be correct in the case of the initial two synthetic heme analogues that we have studied extensively to date, the protoporphyrin chelates of tin and cobalt.

\section{Sn-protoporphyrin: a potent inhibitor of heme degradation}

Sn-protoporphyrin, unlike inorganic tin or the organotin compounds previously studied, did not induce heme oxygenase in liver, kidney, or any other tissue studied. Rather, this synthetic heme analogue proved to be a powerful competitive inhibitor of the enzyme (Fig. 2, and references 27-29), thus considerably diminishing the rate of heme degradation to bile pigments. The compound displayed other distinctive properties as well; after administration of a single small dose to animals, Sn-protoporphyrin $(a)$ produces an immediate $(\sim 30 \mathrm{~min})$ complete heme saturation of hepatic tryptophan pyrrolase, an effect which gradually subsides after 12-16 $\mathrm{h} \mathrm{(30);} \mathrm{(b)} \mathrm{elicits} \mathrm{a} \mathrm{large,} \mathrm{transient}$ ( $\sim 6-12 \mathrm{~h}$ ) output of heme in bile (31), (c) diminishes biliary bilirubin output significantly $(\sim 6-12 \mathrm{~h})(32),(d)$ markedly diminishes, during approximately the same time period, the production of carbon monoxide derived from the catabolism of endogenous as well as exogenous heme (33), and $(e)$ decreases completely the increased output of biliary bilirubin resulting from exogenous heme infusions and partially the increase produced by infusion of heat-damaged erythrocytes (32).

Exogenous heme is metabolized in the liver while the heme derived from heat-damaged erythrocytes is primarily metabolized in the spleen. The exogenous heme infusion data (32) clearly indicate that $\mathrm{Sn}$-protoporphyrin has a major inhibitory action on hepatic heme catabolism. However, the incomplete suppression of biliary bilirubin output, as well as a considerable interanimal variation in response to Sn-protoporphyrin (32), in experiments with heat-damaged erythrocytes leaves unresolved the question of the extent to which this compound can suppress splenic heme catabolism. For example, it is possible that some fraction of the heat-damaged erythrocytes, or their contained heme, may reach the liver undegraded; thus, the observed effect of the metalloporphyrin on putative splenic heme catabolism could in fact represent a composite inhibitory action on both hepatic and splenic heme degradation. At present, therefore, it is uncertain as to what degree Sn-protoporphyrin would inhibit heme degradation resulting from normal erythrocyte senescence, which is a process that takes place primarily in the spleen, in contrast to its effects under circumstances where there is a considerable heme overload presented to the spleen and the liver for catabolism, as in severe hemolytic states. This question is being studied further. No significant effect of the metalloporphyrin on the metabolic disposition of preformed bilirubin has been identified (32) and the action of Sn-protoporphyrin appears to be directed primarily against heme oxygenase.

Extensive toxicological studies in animals have shown Snprotoporphyrin to be quite innocuous. In newborn rats, the

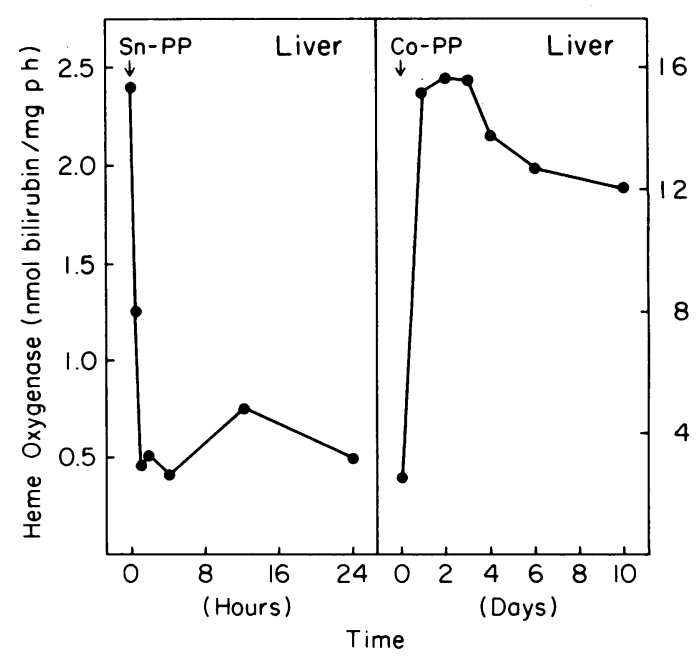

Figure 2. Effect of Sn-protoporphyrin (Sn-PP) and Co-protoporphyrin (Co-PP) when administered once at a dose of $50 \mu \mathrm{mol} / \mathrm{kg}$ body wt on hepatic heme oxygenase activity in the rat. 
therapeutic/toxicity ratio is at least 150:1. In anemic mutant $\left(\mathrm{sph}^{\mathrm{ha}} / \mathrm{sph}^{\mathrm{ha}}\right)$ mice, administration, in divided weekly doses $(100$ $\mu \mathrm{mol} / \mathrm{kg}$ body wt for $32 \mathrm{wk}$ ), of a total amount of Sn-protoporphyrin of $3,200 \mu \mathrm{mol} / \mathrm{kg}$ body wt evoked no toxicity in this biologically fragile strain of genetically anemic animals, while producing a sustained decline in serum bilirubin levels (34). Snprotoporphyrin also suppresses both naturally occurring and experimentally induced jaundice in newborn and adult animals, including experimental primates and humans (Fig. 3, and references 35,36 ). In other studies we have established that the apparent affinity of purified human serum albumin for Sn-protoporphyrin is less than $1 / 1000$ of that for heme and that the synthetic metalloporphyrin exerts no discernible effect on the binding of bilirubin to this serum protein.

Thus Sn-protoporphyrin has a potent ability to suppress the rate of heme catabolism, and through this action, the compound has proved capable of lowering elevated levels of serum bilirubin in animals and humans. The reason why protoporphyrin chelation of tin, unlike the comparable situation with iron or with cobalt (see below), obliterates the heme oxygenase-inducing action of the metal contained in the metal-porphyrin complex and results in the production of a potent in vivo, as well as in vitro, competitive inhibitor of heme oxygenase remains unknown.

It is clear that an innocuous, effective inhibitor of the catabolism of heme to bilirubin would be an agent of considerable therapeutic value in the prevention or treatment of excessive hyperbilirubinemia in certain premature human newborn. The extent to which Sn-protoporphyrin may find use to suppress jaundice in this clinical circumstance will hinge on the identification and elimination of any significant toxic potential of the compound. To this end, the fact that the liver excretes a large part of the heme whose catabolism to bile pigment is inhibited after Sn-protoporphyrin administration (31) is reassuring since this action bears on the question of the possible (though yet undefined) risks to the premature infant of heme transiently retained in the liver compared with the known risks of bilirubin deposition in the brain in excessively jaundiced premature in-

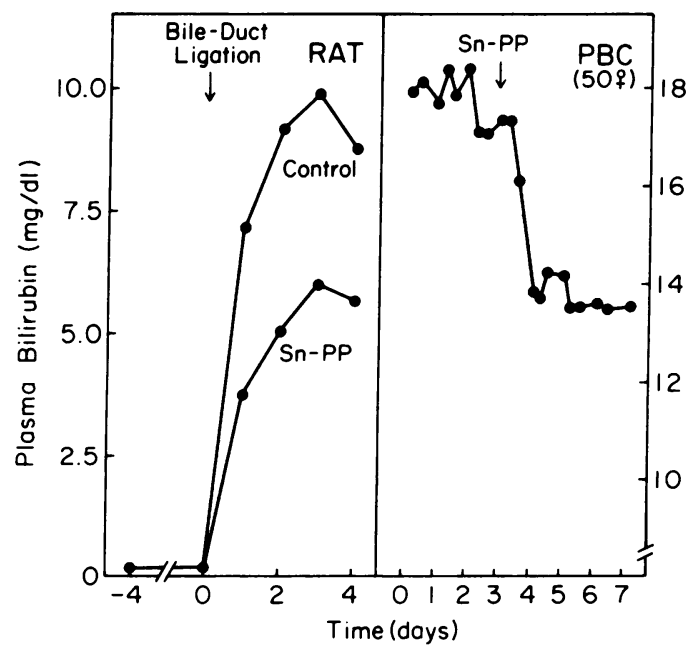

Figure 3. Effect of Sn-protoporphyrin (Sn-PP) $(100 \mu \mathrm{mol} / \mathrm{kg}$ body wt) administered at $4 \mathrm{~d}$ before and on the day of surgery on hyperbilirubinemia in the bile-duct-ligated rat. Effect of Sn-PP $(2 \times 0.25 \mu \mathrm{mol} / \mathrm{kg}$ body wt) on the levels of serum bilirubin in a patient with primary biliary cirrhosis (PBC). fants. To date, transient photosensitivity in some subjects, following administration of single doses of the metalloporphyrin exceeding $1.0 \mu \mathrm{mol} / \mathrm{kg}$ body $\mathrm{wt}$, has been the only deleterious action of the compound that we have identified in over 120 studies in adults.

\section{Co-protoporphyrin: a potent and long-acting inducer of heme oxygenase}

If inorganic tin and Sn-protoporphyrin represent models of metallic species whose effects on heme degradation are in diametric opposition, inorganic cobalt and Co-protoporphyrin constitute a paradigm of those elements whose biological actions in their inorganic form are, in contrast, greatly magnified by protoporphyrin chelation. Inorganic cobalt in a single dose produces an induction of hepatic heme oxygenase, which generally peaks at $\sim 16 \mathrm{~h}$ and subsides by $\sim 48-72 \mathrm{~h}$; this is associated with a concurrent decline in cytochrome P-450 levels and transient lowering, followed by a "rebound" increase, of ALA-synthase activity to above normal levels before the entire response ends $(16,37)$. In contrast, a single equimolar dose of Co-protoporphyrin produces an induction response in hepatic heme oxygenase that is substantially greater in degree. This response is also sustained (Fig. 2) for a much longer period of time; it persists for as long as 3-5 wk in treated animals. A pronounced decline in ALA-synthase activity accompanies this response, and as a consequence of these dual and prolonged actions on heme synthesis and degradation, a profound decrease in cytochrome P450 levels occurs (38). Periodic administration of Co-protoporphyrin (e.g., six doses over a 3-mo period; Fig. 4) leads to a sustained, very high level of hepatic heme oxygenase activity, and because of this enzymatic response and the persistent decline in ALA-synthase activity noted above, a comparably prolonged and intense decline $(\sim 85 \%)$ of cytochrome P-450 content of liver cells occurs. The decline in hepatic cytochrome P-450 content is manifest not only by the expected decrease in cellular heme content, but also by decreases in apocytochrome P-450 moieties in endoplasmic reticulum membranes, as well as by comparable decreases in the oxidative biotransformations of various chemicals that are dependent on this hemeprotein (17).

Co-protoporphyrin, like Sn-protoporphyrin, cannot be enzymatically degraded to bile pigments because neither metalloporphyrin binds molecular oxygen. Apart from this similarity and the fact that their contained metals are, in their inorganic forms, both potent inducers of heme oxygenase, an explanation of why one of these two synthetic metalloporphyrins, Sn-protoporphyrin, potently inhibits heme oxygenase, while the other, Co-protoporphyrin, markedly induces the same enzyme remains unclear. There may be some clue to this riddle in the fact that in vitro, Co-protoporphyrin, like Sn-protoporphyrin, also acts as a competitive inhibitor of heme oxygenase (28); but in vivo, the intense enzyme-inducing action of the metalloporphyrin becomes the principal expression of its biological properties (38). Therefore, whether Sn-protoporphyrin also has dual actions on heme oxygenase, with the inhibitory action dominating in vivo, is a potentially important question because if the answer is affirmative, it may prove that many synthetic heme analogues possess both inducing and inhibitory properties for heme oxygenase and that the biological action which is ultimately expressed in vivo is dependent principally on the nature of the central metal atom of the metal-porphyrin chelate.

The intense and sustained induction of heme oxygenase, coupled with the pronounced depletion of cellular cytochrome 


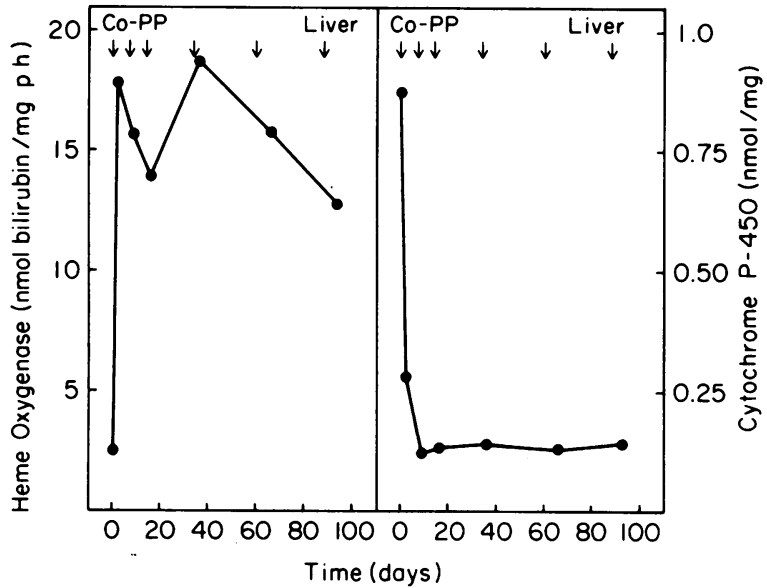

Figure 4. Effect of periodic administration of Co-protoporphyrin (CoPP) (six injections of $50 \mu \mathrm{mol} / \mathrm{kg}$ body wt at the times indicated by arrows) on heme oxygenase activity and cytochrome P-450 content in liver of the rat.

P-450, evoked by Co-protoporphyrin in single or multiple doses (Fig. 4) would be expected to elicit marked metabolic aberrations in the host animal. Such aberrations occur and include not only a major impairment of cytochrome P-450-dependent biotransformations, but also striking endocrine dysfunction whose relationship to cytochrome P-450 metabolism is not yet fully elucidated. For example, within a week of the administration of two doses of Co-protoporphyrin in rats, serum testosterone (T), $T_{3}$, and $T_{4}$ levels decline markedly, without reciprocal increases in luteinizing hormone and thyroid-stimulating hormone. These endocrine alterations, which are not produced by equivalent doses of inorganic cobalt, heme, or a number of other synthetic metalloporphyrins, suggest an action of Co-protoporphyrin directly, or perhaps indirectly through its derangement of tissue cytochrome P-450 content and function, on some endocrine regulatory site(s) within the hypothalamic-pituitary axis. The endocrine-metabolic aberrations gradually recede after the administration of Co-protoporphyrin has been terminated, and heme oxygenase activity and tissue cytochrome $\mathrm{P}-450$ content revert to normal.

The ability to markedly deplete, on a long-term basis, tissue content of cytochrome $\mathrm{P}-450$ with Co-protoporphyrin represents a novel property of this synthetic heme analogue. This biological property is likely to prove useful experimentally in that it may provide information of clinical value. For example, the endocrine aberrations produced by Co-protoporphyrin suggest that it may be possible, by substantially diminishing cytochrome $P-450$ levels in the liver and other tissues, to define further aspects of the role(s) that this hemeprotein normally plays in metabolic homeostasis. These physiological functions of cytochrome P-450, in contrast with the functions of this hemeprotein in pharmacological or toxicological contexts, have by no means been entirely clarified. By the use of this metalloporphyrin as well as other agents that induce cytochrome $\mathrm{P}-450$, it will also be possible to assess the metabolic characteristics of individual animals during periods of enhanced or diminished cytochrome $P-450$ function, thus obviating genetic variables in such studies. Experiments along these lines may help to define the biological advantages or disadvantages of circumstances in which the catalytic activities of cytochrome P-450 are either elevated or decreased by nutritional status, the use of certain drugs, or other factors.

Finally, it will not only be possible, in animals markedly depleted of their tissue cytochrome P-450 content, to study which isozymes of this class of hemeproteins are most readily degraded or are most rapidly resynthesized following the administration of hormones, drugs, or toxic foreign chemicals, but by comparing the properties of parent drugs, putative carcinogens, and other biologically active chemicals in animals with low levels of hepatic cytochrome P-450 and in animals with high levels of the hemeprotein, it may be possible to determine the extent to which oxidative metabolism, involving cytochrome $\mathrm{P}-450$, is an essential determinant of the biological actions associated with the parent substance in question. Thus, for example, the question of whether a metabolite of a particular drug, or the untransformed precursor compound itself, is the biologically active agent in vivo can be more readily addressed than is presently the case. The availability of this type of information would be of considerable importance in defining the nature of the proximately active chemical species in the field of therapeutics. Such information would also have value in facilitating specific drug design within those classes of therapeutic agents that undergo extensive oxidative metabolism to other derivatives in vivo, since many metabolites of drugs have biological properties that mimic, or may even be distinct from, those of the parent compound. If those drugs that yield active metabolites following their in vivo biotransformation could be readily distinguished from those that do not, such information would represent a significant advance in clinical therapeutics.

\section{Summary}

Studies with synthetic metal-porphyrin complexes in which the central iron atom of heme is replaced by other elements indicate that those heme analogues that cannot be enzymatically degraded to bile pigments possess novel biological properties that may have considerable clinical as well as experimental value. Such studies have revealed the important role that the central metal atom plays in determining the physiological and pharmacological properties of metal-porphyrin complexes; and they have demonstrated that the form in which animals and humans are exposed to trace metals, i.e., inorganic, organified, porphyrin-chelated, etc., can be of great importance in determining the biological responses that such elements elicit, especially with respect to actions on heme synthesis and degradation and cytochrome P-450 formation and function. Study of the biological properties of synthetic metalloporphyrins represents a potentially fruitful area of research and the results may have significant value for basic as well as clinical disciplines.

\section{Acknowledgments}

This research was supported by U. S. Public Health Service grant ESO1055 and grants from the Mobil Corp., the Ogden Corp., the Renfield Foundation, and the Dyson Foundation.

\section{References}

1. Calvin, M. 1969. Chemical Evolution. Molecular Evolution Towards the Origin of Living Systems on the Earth and Elsewhere. Oxford University Press, New York. 9-26. 
2. Kappas, A., S. Sassa, and K. E. Anderson. 1982. In The Metabolic Basis of Inherited Diseases. J. B. Stanbury, J. B. Wyngaarden, D. S. Fredrickson, J. L. Goldstein, and M. S. Brown, editors. McGraw-Hill Book Co., New York. 1301-1384.

3. Conney, A. H. 1967. Pharmacol. Rev. 19:317-366.

4. Vessel, E. S. 1984. Ann. NY. Acad. Sci. 428:293-307.

5. Alvares, A. P., and A. Kappas. 1977. J. Biol. Chem. 252:63736378.

6. Nebert, D. W., and H. V. Gelboin. 1968. J. Biol. Chem. 243: 6242-6249.

7. Granick, S., and A. Kappas. 1967. J. Biol. Chem. 242:4587-4593.

8. Kappas, A. 1968. N. Engl. J. Med. 278:378-384.

9. Kappas, A., and S. Granick. 1968. J. Biol. Chem. 243:346-351.

10. Sassa, S., H. L. Bradlow, and A. Kappas. 1979. J. Biol. Chem. 254:10011-10020.

11. Smith, T. J., G. S. Drummond, I. A. Kourides, and A. Kappas. 1982. Proc. Natl. Acad. Sci. USA. 79:7537-7541.

12. Alvares, A. P., K. E. Anderson, A. H. Conney, and A. Kappas. 1976. Proc. Natl. Acad. Sci. USA. 73:2501-2504.

13. Kappas, A., K. E. Anderson, A. H. Conney, and A. P. Alvares. 1976. Clin. Pharmacol. Ther. 20:643-653.

14. Anderson, K. E., A. H. Conney, and A. Kappas. 1982. Nutr. Rev. 40:161-171.

15. Kappas, A. 1983. In Polycyclic Aromatic Hydrocarbons: Evaluation of Sources and Effects. National Academy Press, Washington, DC. 1-76.

16. Maines, M. D., and A. Kappas. 1974. Proc. Natl. Acad. Sci. USA. 71:4293-4297.

17. Kappas, A., and G. S. Drummond. 1984. Environ. Health Perspect. 57:301-306.

18. Kappas, A., and M. D. Maines. 1976. Science (Wash. DC). 192: 60-62.

19. Goldwasser, E., L. O. Jacobson, W. Friend, and L. Plzak. 1957. Science (Wash. DC). 125:1085-1086.

20. Isom, G., and J. L. Way. 1973. Toxicol. Appl. Pharmacol. 24: 449-456.

21. Hodnett, E. M., and W. J. Dunn III. 1972. J. Med. Chem. 15: 339.
22. O'Hara, G. P., D. E. Mann, and R. F. Gautieri. 1971. J. Pharm. Sci. 60:473-474.

23. U. S. Dept. of Health, Education and Welfare. 1976. Criteria for a Recommended Standard . . . Occupational Exposure to Organotin Compounds. National Institute for Occupational Safety and Health, Washington, DC.

24. van der Kerk, G. J. M. 1976. In Organotin Compounds: New Chemistry and Applications. J. J. Zuckerman, editor. American Chemical Society, Washington, DC. 1.

25. Rosenberg, D. W., G. S. Drummond, H. C. Cornish, and A. Kappas. 1980. Biochem. J. 190:465-468.

26. Rosenberg, D. W., G. S. Drummond, and A. Kappas. 1982. Mol. Pharmacol. 21:150-158.

27. Drummond, G. S., and A. Kappas. 1981. Proc. Natl. Acad. Sci. USA. 78:6466-6470.

28. Yoshinaga, T., S. Sassa, and A. Kappas. 1982. J. Biol. Chem. 257:7778-7785.

29. Drummond, G. S., and A. Kappas. 1982. Science (Wash. DC). 217:1250-1252.

30. Kappas, A., G. S. Drummond, and M. K. Sardana. J. Clin. Invest. 75:302-305.

31. Kappas, A., C. S. Simionatto, G. S. Drummond, S. Sassa, and K. E. Anderson. 1985. Proc. Natl. Acad. Sci. USA. 82:869-900.

32. Simionatto, C. S., K. E. Anderson, G. S. Drummond, and A. Kappas. 1985. J. Clin. Invest. 75:513-521.

33. Milleville, G. S., M. D. Levitt, and R. R. Engel. 1985. Pediatr. Res. 19-94-96.

34. Sassa, S., G. S. Drummond, S. E. Bernstein, and A. Kappas. 1985. J. Exp. Med. 162:864-876.

35. Cornelius, C. E., and P. A. Rodgers. 1984. Pediatr. Res. 18:728730.

36. Kappas, A., G. S. Drummond, C. S. Simionatto, and K. E. Anderson. 1984. Hepatology (Baltimore). 4:336-341.

37. Maines, M. D., and A. Kappas. 1975. J. Biol. Chem. 250:41714177.

38. Drummond, G. S., and A. Kappas. 1982. Proc. Natl. Acad. Sci. USA. 79:2384-2388. 